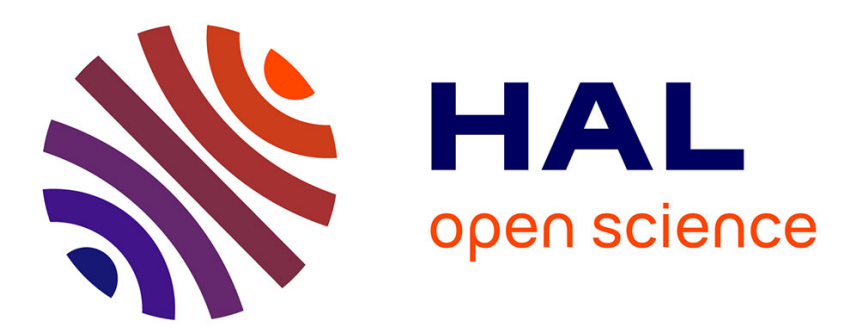

\title{
Determination of the index profile in a graded index optical waveguide: a comparaison between perturbation method and W.K.B. analysis and chemical analysis
}

A. Guez, P.C. Jaussaud, G.H. Chartier

\section{- To cite this version:}

A. Guez, P.C. Jaussaud, G.H. Chartier. Determination of the index profile in a graded index optical waveguide: a comparaison between perturbation method and W.K.B. analysis and chemical analysis. Revue de Physique Appliquée, 1979, 14 (10), pp.847-852. 10.1051/rphysap:019790014010084700 . jpa-00244671

\section{HAL Id: jpa-00244671 \\ https://hal.science/jpa-00244671}

Submitted on 1 Jan 1979

HAL is a multi-disciplinary open access archive for the deposit and dissemination of scientific research documents, whether they are published or not. The documents may come from teaching and research institutions in France or abroad, or from public or private research centers.
L'archive ouverte pluridisciplinaire HAL, est destinée au dépôt et à la diffusion de documents scientifiques de niveau recherche, publiés ou non, émanant des établissements d'enseignement et de recherche français ou étrangers, des laboratoires publics ou privés. 


\title{
REVUE DE PHYSIQUE APPLIQUÉE
}

Classification

Physics Abstracts

$42.10-42.80-42.82$

\section{Determination of the index profile in a graded index optical waveguide : a comparaison between perturbation method and W.K.B. analysis and chemical analysis}

\author{
A. Guez, P. C. Jaussaud and G. H. Chartier \\ Laboratoire de Génie Physique, Greco C.N.R.S. Micro-Ondes, \\ E.N.S.I.E.G., B.P. no 46, 38402 St Martin d'Hères, France \\ (Reçu le 12 février 1979, révisé le 13 juin 1979, accepté le 20 juin 1979)
}

\begin{abstract}
Résumé. - Disposer d'une méthode non destructive de caractérisation d'un guide d'ondes lumineuses est indispensable. Nous comparons ici deux méthodes différentes qui peuvent être utilisées à cette fin, et expliquons pourquoi elles sont complémentaires. La validité de ces méthodes est testée par comparaison avec une analyse à la microsonde électronique à balayage.
\end{abstract}

Abstract. - The use of a non destructive method for determining the index profile of a waveguide is imperative. We compare here 2 different methods that can be used for this purpose, show a very good agreement between the methods, and explain why they are complementary.

1. Introduction. - When an optical waveguide has been fabricated, it is important to caracterize it by non destructive means. A considerable amount of work has been done during the last five years, mainly for graded index optical waveguides, and several methods have been proposed to determine the refractive index profile (R.I.P.) of planar optical waveguides.

- interferometric measurements have been used $[1,2,3]$, but these measurements need thin slabs (less than $100 \mu \mathrm{m}$ ) to be cut in the sample and is thus partly destructive ;

- electron microprobe can be used to find the ionic concentration which induces the gradient index $[3,4]$. This method cannot be used for all ions, and needs the relationship between index variation and ionic concentration to be known. Furthermore, because of the finite size of the cross section of the probing electron beam, the first two microns of the waveguide cannot be analysed :

- angular dependance of reflectivity has recently been developped [5], and is shown to be non destructive only in the case of rapidly varying profiles :

- several methods have also been used which compute the index profile from the mode spectrum of the guided light;

In a first kind of methods, the shape of the profile is assumed (for example gaussian) and from reduced curves the caracteristic parameters of the R.I.P. are deduced (maximum index variation, depth). These methods also include the computing of the spectrum assuming a given shape, and then the comparison of the computed spectrum with the experimental one. Numerical iterations have to be used to find a realistic profile [6].

In a second kind of methods, the shape of the R.I.P. is directly determined from the experimental mode spectrum. These methods use the formal analogy between the Schrödinger wave equation of quantum mechanics and the propagation equation of a TE mode in a planar waveguide.

(i) W.K.B. method was first used in a direct way to find the mode spectrum of a given R.I.P. [7]. Following Gedeon's conclusions, we shall use direct W.K.B. results as exact results for the mode spectrum of a given R.I.P. It has also been used to have an idea of how the R.I.P. starts, near the surface [8]. Most laboratories now use the so called inverse W.K.B. method (I.W.K.B.), first presented by White and Heidrich (1976) [9]. Assuming the profile is formed of successive linear segments, I.W.K.B. can give a good estimate of the R.I.P., provided the index function is monotonically decreasing from the surface, and provided the surface index is known. This last restriction may induce an error on the beginning of the profile, but has no consequence on the determination of the rest of the profile, which is quite stable. 
(ii) Perturbation analysis (P.A.) was developped for gaussian and complementary error function profiles $[10,11,12]$. It was shown in these papers, that perturbation method gives good results for the region close to the surface, as could be expected from its principles.

In the present paper we shall compare :

a) the R.I.P. obtained from a given mode spectrum by I.W.K.B. and P.A. As the mode spectrum is obtained from a given theoretical profile, it is then possible to compare the accuracy of the methods ;

b) the R.I.P. obtained from their mode spectrum for some of our samples to a chemical analysis of these samples with a scanning electron microscope.

2. Definitions. - Let the profile function be $n(x), n(0)$ being a maximum of $n(x)$ (Fig. 1). Assume a transverse electric mode in the guide, of the form and

$$
E_{y}(x, y, z, t)=E_{0}(x) \exp \left(j\left(\omega t-\beta_{m} z\right)\right),
$$

$$
\omega=c k, \quad k=\frac{2 \pi}{\lambda}
$$

is the free space propagation constant, and $\beta_{m}$ are the guide propagation constants. In this case, the wave equation in the guide is

$$
\frac{\mathrm{d}^{2} E_{0}}{\mathrm{~d} x^{2}}+\left(k^{2} n^{2}(x)-\beta^{2}\right) E_{0}(x)=0 .
$$

Let $n_{m}=\beta_{m} / k$ be the effective index of the $m$ th mode of the guide.

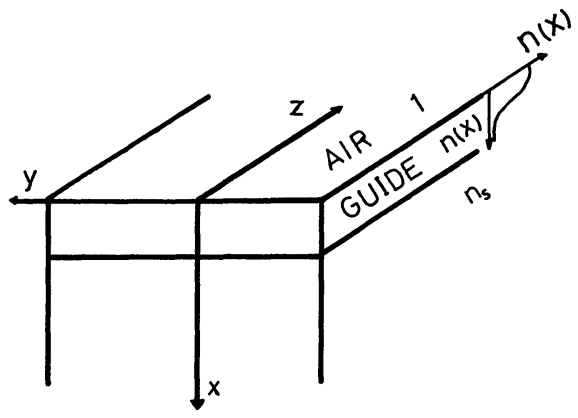

Fig. 1. - Schematical configuration of a waveguide structure.
3. W.K.B. method. - In this well known method, developped for quantum mechanics purposes, it is shown that

$$
k \int_{0}^{x_{m}} \sqrt{n^{2}(x)-n_{m}^{2}} \mathrm{~d} x=m \pi+\frac{\pi}{4}+\varphi_{m}(0)
$$

where

$$
\varphi_{m}(0)=\operatorname{Arctg} \sqrt{\frac{n_{m}^{2}-n_{\mathrm{ex}}^{2}}{n^{2}(0)-n_{m}^{2}}} .
$$

$x_{m}$ is the turning point of the wave function $E_{0}$, i.e. the point where $\mathrm{d}^{2} E_{0} / \mathrm{d} x^{2}=0$, so that at $x_{m}$

$$
n\left(x_{m}\right)=\beta_{m} / k .
$$

Given $n(x)$, a computed solution can be found for (2), that leads to $n_{m}$. Comparison between this solution and a complete solution of (1) was first done by Gedeon [7] and agreement was found to be better than 0.00015 , value that we find too.

We shall thus assume that direct W.K.B. method gives exact values for $n_{m}$.

4. Inverse W.K.B. method. - This method [9] is valid for the case where $n(x)$ has its maximum at $x=0$.

Furthermore it is assumed that air-guide separation is perfectly reflective, so that $\varphi_{m}(0)=\pi / 2$.

It is then assumed that the index profile is given by a set of linear segments so that

$$
n(x)=n_{m}+\frac{n_{m-1}-n_{m}}{x_{m}-x_{m-1}}\left(x_{m}-x\right) \text { for } x_{m-1}<x<x_{m} .
$$

If reduced coordinate $z=x / \lambda$ is used, one thus can get for $M$ modes

$$
\int_{0}^{z_{m}} \sqrt{n^{2}(z)-n_{m}^{2}} \mathrm{~d} z=\frac{4 m-1}{8} \quad m=1,2, \ldots, M
$$

and using (3), it can be shown that

$$
\begin{aligned}
z_{m}=z_{m-1}+\frac{3}{2}\left(\frac{n_{m-1}+3 n_{m}}{2}\right)^{-1 / 2}\left(n_{m-1}-n_{m}\right)\left[\frac{4 m-1}{8}\right]-\frac{2}{3} \sum_{k=1}^{m-1}\left(\frac{n_{k-1}-n_{k}}{2}+n_{m}\right)^{1 / 2} \times \\
\times\left(\frac{z_{k}-z_{k-1}}{n_{k-1}-n_{k}}\right)\left[\left(n_{k-1}-n_{m}\right)^{3 / 2}-\left(n_{k}-n_{m}\right)^{3 / 2}\right] \text { for } m=2,3, \ldots, M
\end{aligned}
$$

and

$$
z_{1}=\frac{9}{16}\left(\frac{n(0)+3 n_{1}}{2}\right)^{-1 / 2}\left(n_{0}-n_{1}\right)^{-1 / 2} .
$$


These results give the $x$ values where $n(x)=n_{m}$ and then an approximate shape for the index profile, with the restriction that one must postulate the value for $n(0)$.

5. Perturbation method. - 5.1 No eVANESCENT FIELD OCCURS. - Let the index profile be of the form : $n(x)=n_{1}-\Delta n f(x)$. Then (1) becomes :

$$
\begin{aligned}
& \frac{\mathrm{d} E_{0}}{\mathrm{~d} x^{2}}+\left[k^{2} n_{1}^{2}-\beta^{2}-2 n_{1} k^{2} \times\right. \\
& \left.\quad \times \Delta n f(x)+k^{2} \Delta n^{2} f^{2}(x)\right] E_{0}=0 .
\end{aligned}
$$

Thus the problem we have to solve is formally identical to that of a quantum particle of mass $m$ such that $2 m / \hbar^{2}=1$, of energy $k^{2} n_{1}^{2}-\beta^{2}$, in a potential well $V(x)=2 n_{1} k^{2} \Delta n f(x)-k^{2} \Delta n^{2}(x)$ for $x>0$ and $V(x)=k^{2}\left(n_{1}^{2}-n^{2}\right.$ ext) for $x<0$. The solution of this problem is well known in some cases if field penetration in air can be neglected, i.e. if $V(x)$ is large for $x<0$. Among these we shall consider 2 cases :

$$
\begin{array}{ll}
\text { (i) } f(x)=\left(\frac{x}{a}\right)^{2} & x>0, f(x)=\infty \quad x<0 \\
\text { (ii) } f(x)=\frac{x}{a} & x>0, f(x)=\infty \quad x<0
\end{array}
$$

where $a$ can be defined as the guide depth.

For (i) case it is shown in [11] that, if $\Delta n^{2}$ term is neglected

$$
\left(\frac{\beta_{p}}{k}\right)^{2}=n_{1}^{2}-(4 p+3) \frac{\sqrt{2 n_{1} \Delta n}}{k a}
$$

and $E_{0}(x)=A \mathrm{e}^{-Q^{2} / 2} H_{2 p+1}(Q)$, where

$$
Q=\frac{x}{\alpha}, \quad \alpha=\left(\frac{a^{2}}{2 n_{1} \Delta n}\right)^{1 / 4} \text { and } H_{2 p+1}
$$

is the hermitian polynomial order $2 p+1$ and for (ii), it is found that

$$
\left(\frac{\beta_{p}}{k}\right)^{2}=n_{1}^{2}-\left(\frac{2 n_{1} \Delta n}{k a}\right)^{2 / 3} \lambda_{p}
$$

where $\lambda_{p}$ is the $p$ th zero of the Airy function [11] which gives the electric field

$$
\begin{aligned}
E(x)=A\left(\alpha^{1 / 3} X\right)^{1 / 2} \times & \\
& \times\left[J_{1 / 3}\left(\frac{2}{3} \alpha^{1 / 2} X^{3 / 2}\right)+J_{-1 / 3}\left(\frac{2}{3} \alpha^{1 / 2} X^{3 / 2}\right)\right]
\end{aligned}
$$

where

$\alpha=\frac{2 n_{1} \Delta n k^{2}}{a}, \quad X=\left(n_{1}^{2} k^{2}-\beta^{2}\right) / \alpha-x, \quad X>0$.

For $\mathrm{X}<0$ (i.e. outside of the turning points) $E(x)$ varies as

$$
\left(\alpha^{1 / 3}|X|\right)^{1 / 2} K_{1 / 3}\left(\frac{2}{3} \alpha|X|^{3 / 2}\right),
$$

$J$ and $K$ are Bessels and Hankel's functions.
Then 2 curves are plotted : $\left(\beta_{p} / k\right)^{2}$ versus $p$ or $\lambda_{p}$. A straight line is observed in one of the cases, if the R.I.P. is parabolic or linear from this straight line, the parameters $a$ and $\Delta n$ can be deduced.

If however the R.I.P. is neither parabolic nor linear, but deviates from either law by some term which we will call $\varphi(x)$ then the perturbation theory can be applied to find the correction which has to be substracted from experimental values of $(\beta / k)^{2}$ to get a straight line. For instance, to first order perturbation theory

$\Delta\left[n_{1}^{2}-\frac{\beta^{2}}{k}\right]=-\Delta\left(\frac{\beta}{k}\right)^{2}=\frac{\int_{0}^{\infty} E_{0}(x)^{2} \varphi(x) \mathrm{d} x}{\int_{0}^{\infty} E_{0}(x)^{2} \mathrm{~d} x}$

first and second order perturbation theory have to be applied for the gaussian case, and complete results have been presented in a previous paper [12]. The same paper presented the first order calculation when an $x^{3}$ term (which occurs in error function) is added to a linear profile. We also calculated the effect on an $a_{2} x^{2}$ term on the linear profile, to get a more general polynomial term. We found for that term

$$
\Delta n_{\text {eff }}^{2}=\frac{2}{15}\left(\frac{2}{\pi}\right)^{5 / 3}\left(n_{1} \Delta n\right)^{1 / 3}\left(\frac{1}{k a}\right)^{4 / 3} a_{2} \lambda^{2} p
$$

Perturbation theory will be used in the following way :

- from the first points, parameters $a$ and $\Delta n$ are calculated using formulae (8) or (9) ;

- with these parameters, corrections for gaussian, erfc $(x / a)$ etc... functions are calculated. This correction is substrated from the experimental values of $(\beta / k)^{2}$. If the corrected values are aligned with the first modes values, it may be inferred that the right profile has been found. If not, another type of perturbation has to be tested.

All these effects are calculated assuming that the unperturbed R.I.P. is bound by a hard wall at $x=0$, i.e. that no evanescent field is allowed in air, which is obviously wrong. Using the Wronskian method we had calculated the effect of evanescent field on the propagation constant [12] but we had not estimated the accuracy of this calculation. We will thus compare here this calculated correction to its exact value.

5.2 EFFECT OF FIELD PENETRATION IN AIR. - For sake of brevity and simplicity we shall here only study this effect for a linear profile, although similar results have been obtained for parabolic profiles.

For a linear R.I.P. the effect of the evanescent field was found to be [12]

$$
n_{\mathrm{eff}}^{2}=\frac{1}{\sqrt{n_{1}^{2}-1}} \frac{2 n_{1} \Delta n}{a k} .
$$


For $n_{1}=1.6120$,

$\Delta n=10^{-1} \quad a=16.21 \mu \mathrm{m} \quad$ and $k=9.929 \mu \mathrm{m}^{-1}$

(He-Ne laser) we calculated $\Delta n_{\text {eff }}^{2}=15.8 \times 10^{-4}$.

On the other hand, using direct W.K.B. method, we calculated $(\beta / k)^{2}$ when the evanescent field does not exist $\left(\varphi_{m}(0)=\pi / 2\right)$ and when it does. The difference for the 18 modes of the profile was found to be $\Delta n_{\text {eff }}^{2}=(16.0 \pm 0.2) \times 10^{-4}$.

So even when $\Delta n_{\text {eff }}^{2}$ is large compared to experimental errors, (13) gives a good result.

When (13) gives results that are not negligible compared to experimental errors, this value must be substracted from experimental values to get a second order approximation of $a$ and $\Delta n$ by plotting $(\beta / k)^{2}$ versus $\lambda_{p}$.

6. Comparison between I.W.K.B. and P.A. methods. - We shall proceed as follows :

(i) given the theoretical R.I.P. we calculate the mode spectrum using direct W.K.B. method ;

(ii) starting from the set of values of effective indices $n_{m}=\beta_{m} / k$ and using perturbation analysis we find a P.A. new profile ;

(iii) applying I.W.K.B. to the effective indices, a profile is deduced.

6.1 Gaussian R.I.P. - Figure 2 represents profiles of (i), (ii), (iii) for a typical case values

$$
n(x)=n_{\mathrm{s}}+\Delta n \exp \left(-x^{2} / 2 a^{2}\right),
$$

with

$$
n_{\mathrm{s}}=1.5120, \Delta n=10^{-2}, \quad 2 a^{2}=100 \mu \mathrm{m}^{2},
$$

which are typical parameters for $\mathrm{Li}^{+} \leftrightarrow \mathrm{Na}^{+}$exchanged waveguides in glass.

P.A. analysis gave a gaussian R.I.P. having $\Delta n=9.91 \times 10^{-3}, 2 a^{2}=109 \mu \mathrm{m}^{2}$ so that the errors on $\Delta n$ and a where about $1 \%$ and $4 \%$ respectively.

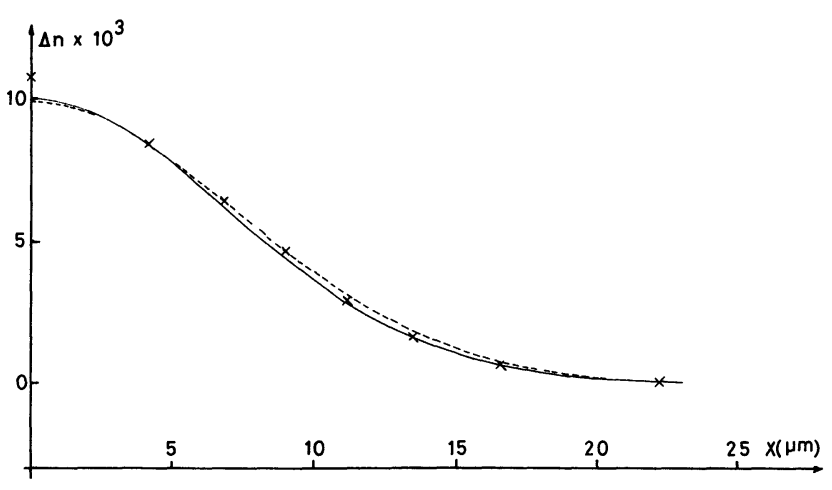

Fig. 2. - Gaussian profile : continuous curve is the given profile, dashed curve the estimated profile from perturbation theory and crosses the points obtained from inverse W.K.B. method.
6.2 COMPLEMENTARY ERROR FUNCTION R.I.P. In the case where $n(x)=n_{\mathrm{s}}+\Delta n \operatorname{erfc}(x / a)$ figure 3 represents the results obtained using (i), (ii), (iii) stages in the case of a theoretical given R.I.P. with $n_{\mathrm{s}}=1.5120, \Delta n=10^{-1}, a=30 \mu \mathrm{m}$, typical values for $\mathrm{Ag}^{+} \leftrightarrow \mathrm{Na}^{+}$exchanged waveguide in glass.

Difference between I.W.K.B. profile and initial profile was of the order of magnitude of the accuracy of direct W.K.B. method $\left(\sim 10^{-5}\right)$. On the other hand, P.A. method gave $n(x)=n_{\mathrm{s}}+\Delta n \operatorname{erfc}(x / a)$ with $\Delta n=1.003 \times 10^{-2}$ and $a=28.8 \mu \mathrm{m}$ so that the accuracy of theses results are $0.3 \%$ and $0.4 \%$ respectively.

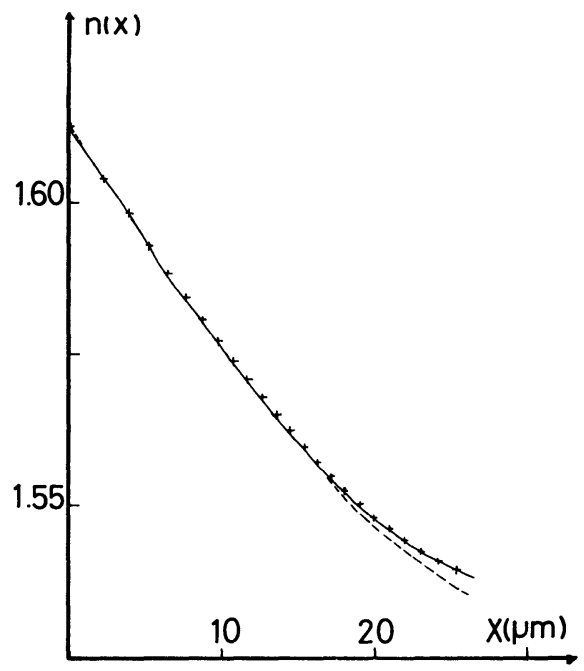

Fig. 3. - Complementary error function : continuous curve is the given erfc profile, dashed curve the estimated profile from perturbation theory and crosses the points obtained from inverse W.K.B. method.

6.3 Stability of THE anAlysis. - If different measurements are performed on the same sample, will they agree each other? This is the problem of stability. White and Heidrich tested this stability by introducing some change $\delta n_{\text {eff }}$ in the values of $n_{\text {eff }}=\beta / k$. They showed that the $x$ value for which $n(x)=\beta / k$ is shifted of $\delta x$ such that $\delta x / x \approx \delta n_{\text {eff }} / n_{\text {eff }}$. We shall thus only examine here the stability of P.A. method.

Let us take into account measurement errors in the curve $(\beta / k)^{2}$ versus $p$ or $\lambda_{\mathrm{p}}$. An error then occurs on $n_{1}$, which can be estimated from (8) or (9). As $\Delta n$ is usually smaller than $0.1, k \sim 10 \mu \mathrm{m}^{-1}$, we roughly have

$$
\frac{\delta n_{1}}{n_{1}} \approx \frac{\delta n_{\mathrm{eff}}}{n_{\mathrm{eff}}}, \text { and } \frac{\delta(\Delta n)}{\Delta n}=\frac{\delta n_{\mathrm{eff}} / n_{\mathrm{eff}}}{\Delta n / n_{1}} .
$$

Usually

$\frac{\delta n_{\text {eff }}}{n_{\text {eff }}} \approx 2 \times 10^{-4}, \frac{\Delta n}{n_{1}} \approx 6 \times 10^{-2}$ to $6 \times 10^{-3}$ 
for ion exchanged waveguides, so that

$$
\frac{\delta(\Delta n)}{\Delta n} \sim 0.3 \% \text { to } 3 \%
$$

Actually, the main difficulty which we meet when using P.A. method is rather to find the real type of function we must use. This implies the knowledge of a large variety of perturbations, which one cannot always easily calculate. This restriction does not occur for I.W.K.B. method.

7. Comparison with experiments. - Experimental situations involve a large number of types of profiles. We shall examine here 2 different types of experiment :

$7.1 \mathrm{Li}^{+}$EXCHANGED WAVEGUIDES. - $\mathrm{a} \mathrm{Li}^{+}$waveguide was fabricated by ion exchange from a eutectic mixture of $\mathrm{Li}_{2} \mathrm{SO}_{4}, \mathrm{~K}_{2} \mathrm{SO}_{4}$, at $T=580^{\circ} \mathrm{C}$, during $t=5 \mathrm{~min}$. The figure 4 shows I.W.K.B. profile (crosses) and P.A. profile (continuous curve). The mode spectrum could only be explained with the following law

$$
n(x)=n_{1}-\Delta n \frac{x}{a_{1}}-\Delta n \frac{x^{2}}{a_{2}^{2}}
$$

where $n_{1}=1.5208, \Delta n=9.2 \times 10^{-3}, a_{1}=64 \mu \mathrm{m}$, $a_{2}=158 \mu \mathrm{m}$, which fits I.W.K.B. also.

No comparison could be done to $\left[\mathrm{Li}^{+}\right]$in the glass using a scanning electron microscope, as $\mathrm{Li}^{+}$is a too light element.

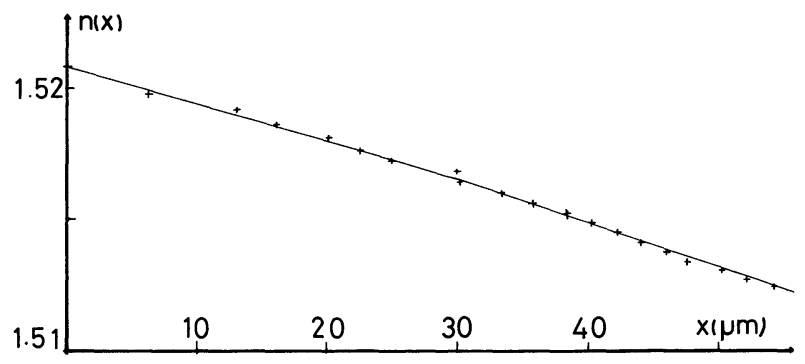

Fig. 4. - Polynomial profile : continuous curve is the estimated profile from perturbation theory, and crosses are obtained from inverse W.K.B. method.

$7.2 \mathrm{~K}^{+}$EXCHANGED WAVEGUIDE. - A potassium waveguide was done by ion exchange at $T=398^{\circ} \mathrm{C}$ during $t=69 \mathrm{~h} \mathrm{45}$, from a liquid $\mathrm{KNO}_{3}$ bath. Figure 5 shows :

- the I.W.K.B. profile (crosses),

- the P.A. profile : the continuous straight line is the imperturbed profile i.e. the profile that we would have from the first 4 modes of the spectrum only.

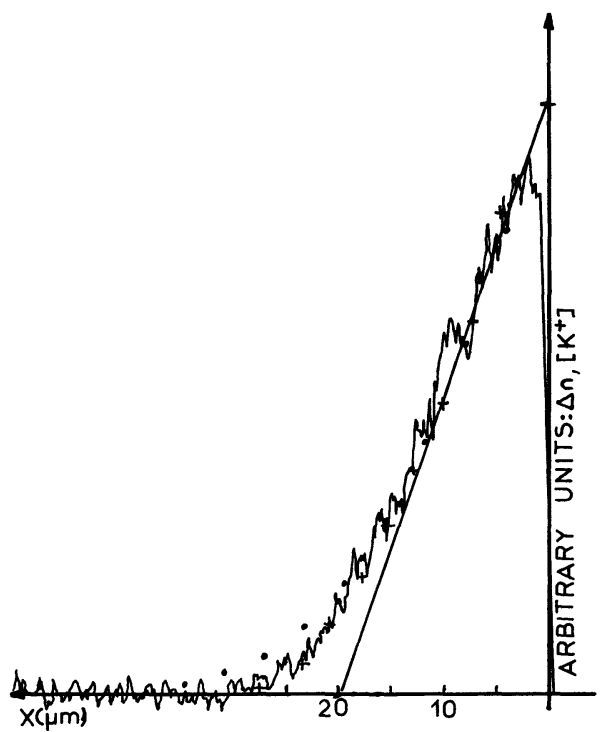

Fig. 5. - Comparison between the index profile and the potassium concentration in the sample, reduced to the same scale.

An erfc $(x / a)$ function had to be used to explain the complete mode spectrum (dots) :

$$
\begin{gathered}
n(x)=n_{\mathrm{s}}+\Delta n \operatorname{erfc}\left(\frac{x}{a}\right), \\
\Delta n=1.12 \times 10^{-2}, \quad a=20.5 \mu \mathrm{m},
\end{gathered}
$$

- the $\left[\mathrm{K}^{+}\right]$concentration in the sample, as measured from a scanning electron microprobe.

8. Conclusion. - Both I.W.K.B. and P.A. method lead to comparable results, which nicely agree with chemical analysis of our samples.

The main advantage of I.W.K.B. is to give the refractive index profile without needing a knowledge of its form. But it needs the value $n_{1}$ at $x=0$. On the other hand P.A. method needs some research to find what corrections on the first approximation have to be introduced to explain the complete mode spectrum. But it easily gives $n_{1}$. In our laboratory we found quite convenient to calculate $n_{1}$ from P.A. method, and then use I.W.K.B. analysis to find the rest of the profile.

This saves some iterations on I.W.K.B. method, and provides a more reliable result for the whole profile.

Acknowledgments. - We would like to thank Mr. Garden, from Laboratoire d'Analyse de Recherches et d'Essais Chimiques (LAREC, ENS-EEG, BP $n^{\circ} 44,38401$ St Martin d'Hères) for performing our scanning electron microprobes measurements. 


\section{References}

[1] Izawa, T. and Nakagome, H., Appl. Phys. Lett. 21 (1972) 584-6.

[2] Martin, W. E., Appl. Opt. 13 (1974) 2112-16.

[3] Stewart, G., Millar, C. A., Laybourn, P. J. R., WilkinSON, C. D. W. and DE LA RUE, R. M., I.E.E.E. J. Quant. Electron. QB 13 (1977) no 4, 192-200.

[4] Giallorenzi, T. G., West, E. J., KiRK, R., Ginther, R. and ANDREws, R. A., Appl. Opt. 12 (1973) 1240-5.

[5] Heibei, J. and Voges, E., IEEE J. Quant. Electron. GE 14 (1978) 501-6.
[6] Conwell, E., Appl. Phys. Lett. 25 (1974) 40-42.

[7] Gedeon, A., Optics Commun. 12 (1974) 329-32.

[8] Vassallo, C., Optical and Quantum Electronics (Chappman and Hall Ltd) 7 (1975) 371-76.

[9] White, J. P., HeidRich, P. F., Appl. Opt. 15 (1976) 151-55.

[10] LotsPeich, J., Opt. Commun. 18 (1976) 567.

[11] Jaussaud, P. C. and Chartier, G. H., J. Phys. D 10 (1977) 645-53.

[12] Ayant, Y., Chartier, G. H. and Jaussaud, P. C., J. Physique 38 (1977) $1089-96$. 